\title{
Parametric-interaction-induced avoided dressed-state crossings in cavity QED: Generation of quantum coherence and equally weighted superposition of Fock states
}

\author{
L. L. Ping $\odot,{ }^{1}$ W. Li $\odot,{ }^{1}$ C. J. Zhu $\odot,{ }^{2, *}$ Y. P. Yang,,${ }^{1 \dagger}$ and G. S. Agarwal ${ }^{3, \ddagger}$ \\ ${ }^{1}$ MOE Key Laboratory of Advanced Micro-Structured Materials, School of Physics Science and Engineering, \\ Tongji University, Shanghai 200092, China \\ ${ }^{2}$ School of Physical Science and Technology, Soochow University, Suzhou 215006, China \\ ${ }^{3}$ Institute for Quantum Science and Engineering and Department of Biological and Agricultural Engineering, \\ Texas A\&M University, College Station, Texas 77843, USA
}

(Received 14 September 2021; accepted 21 December 2021; published 7 January 2022)

\begin{abstract}
We present a paradigm in the field of cavity QED by bringing out remarkable features associated with the avoided crossing of the dressed-state levels of the Jaynes-Cummings model. We demonstrate how the parametric couplings, realized by a second order nonlinearity in the cavity, can turn the crossing of dressed states into avoided crossings. We show how one can generate coherence between the avoided crossing of dressed states. Such coherences result, for example, in quantum beats in the excitation probability of the qubit. The quality of quantum beats can be considerably improved by adiabatically turning on the parametric interaction. We show how these avoided crossings can be used to generate superpositions of even or odd Fock states with the remarkable property of equal weights for the states in superposition. The fidelity of generation is more than $95 \%$. In addition, we show strong entanglement between the cavity field and the qubit with the concurrence parameter exceeding $90 \%$.
\end{abstract}

DOI: 10.1103/PhysRevResearch.4.013014

\section{INTRODUCTION}

Avoided level crossing [1,2] is the phenomenon where two energy levels cannot be pushed through each other and has been an important branch of physics. This phenomenon was first discovered in 1932 by studying a two-level system in quantum mechanics $[3,4]$. Suppose that there are two energy levels labeled by $E_{1}$ and $E_{2}$, respectively. In the absence of external perturbation these two levels would have crossed if the original energy states were degenerate, i.e., $\Delta E=$ $E_{1}-E_{2}=0$. However, in the presence of a perturbation on a two-level system, the energy exchange between two states takes place. Therefore, the eigenvalues of the system will not become degenerate but will have a hyperbolic shape where the minimal energetic distance is proportional to the perturbation strength [5]. The phenomenon of level repulsion is called the avoided level crossing, which has been widely used in many different quantum systems, including atoms [6,7], semiconductor devices [8], and other systems [1,2,9-12], which can be described as a "general" two-state system with some couplings. In cavity QED systems, the avoided level crossing

\footnotetext{
*Corresponding author: chengjie.zhu@yahoo.com

†Corresponding author: yang_yaping@ tongji.edu.cn

‡Corresponding author: girish.agarwal@tamu.edu
}

Published by the American Physical Society under the terms of the Creative Commons Attribution 4.0 International license. Further distribution of this work must maintain attribution to the author(s) and the published article's title, journal citation, and DOI. can be achieved by using the atom-cavity coupling strength. The avoided level crossing then becomes the famous vacuum Rabi splitting [13-19].

In the avoided level crossing region, the two states become strongly mixed, yielding various interesting phenomena. For example, a direct and natural consequence of the avoided crossing is the entanglement behavior [20-23]. This has been exploited to some extent in earlier works that seek to create entangled states such as the $W$ or Greenberger-HorneZeilinger state by using the superpositions of two states that develop at avoided crossings [24-26]. Moreover, the LandauZener tunneling between two energy levels takes place if these two levels of a time-dependent Hamiltonian are avoided crossing [6,27-29]. A Berry phase is accumulated in addition to a dynamical phase if an eigenstate encircles adiabatically degeneracy points [30]. The first-order quantum phase transition, an abrupt change in the ground state of a many-body system as parameters of a system vary, is related with the avoided crossings of two lowest energy levels [28].

In this paper, we present a paradigm in the field of cavity QED by bringing out effects associated with the avoided crossing of the dressed-state levels of the Jaynes-Cummings (JC) model. We demonstrate how the parametric couplings can turn the crossing of dressed states into avoided crossings. Note that this is different from the avoided crossings mentioned earlier in the context of the vacuum Rabi splittings. We show how one can generate coherence between the avoided crossing of dressed states. Such coherences can be monitored via the quantum beats in the excitation probability of the qubit. These avoided crossings can be used to generate superpositions of even or odd Fock states with the remarkable property 


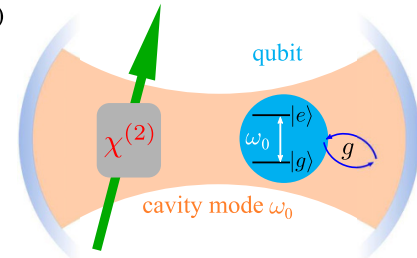

pump field G

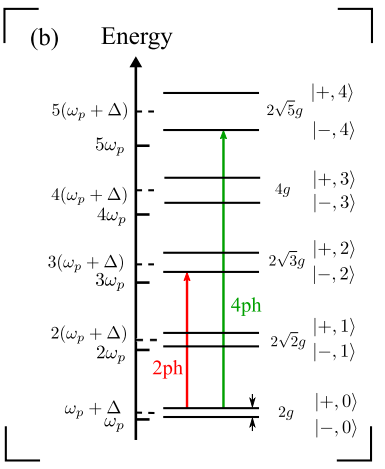

FIG. 1. (a) Schematic diagram of a single qubit-cavity QED system where the cavity photons are generated via the second-order nonlinearity with nonlinear interaction strength $G$ induced by a strong pump field. Here, the qubit transition frequency $\omega_{0}$ is identical to the cavity resonant frequency, and the qubit-cavity coupling strength is denoted by $g$. (b) Dressed-state picture of the system where $| \pm, n\rangle \equiv(|e, n\rangle \pm|g, n+1\rangle) / \sqrt{2}$. The red and green arrows correspond to the transitions involving two- and four-photon processes, respectively.

of equal weights for the states in superposition. In addition, there is strong entanglement between the cavity field and the qubit. The qubit could be an atom or superconducting qubit or a quantum dot. As an example we can produce superpositions of photonic Fock states such as $|0\rangle+i|2\rangle,|0\rangle+i|4\rangle$, and $|1\rangle+i|3\rangle[31]$.

\section{CONVERTING DRESSED LEVELS CROSSING INTO AVOIDED CROSSINGS VIA PARAMETRIC PERTURBATIONS}

To begin with, we consider the standard JC model where a single two-level qubit with transition frequency $\omega_{0}$ is trapped in a single mode cavity with resonant frequency $\omega_{0}$ identical to qubit frequency. In addition as shown in Fig. 1(a), the qubit-cavity coupling strength is denoted by $g$, and the cavity is driven by pumping at frequency $\omega_{P}$, a second-order nonlinear crystal with the nonlinear interaction strength $G$, corresponding to the well-known optical parametric amplification (OPA) process. In a frame rotating with frequency $\omega_{P} / 2$, the Hamiltonian of the system can be written as

$$
H=\Delta\left(a^{\dagger} a+\sigma_{+} \sigma_{-}\right)+g\left(a \sigma_{+}+a^{\dagger} \sigma_{-}\right)+G\left(a^{2}+a^{\dagger 2}\right),
$$

where $a^{\dagger}(a)$ is the creation (annihilation) operator of the cavity mode, and $\sigma_{+}=|e\rangle\langle g|$ and $\sigma_{-}=\sigma_{+}^{\dagger}$ are the spin raising and lowering operators of the qubit. Here, $\Delta=\omega_{0}-\omega_{P} / 2$ is the detunings of the cavity and the qubit with respect to $\omega_{P} / 2$. The structure of the spectrum of the eigenstates of Eq. (1) is discussed in Refs. [32,33]. For many other important outcomes of Eq. (1) in different contexts, see Refs. [34-42]. Obviously, in the absence of the driving field, i.e., $G=0$, the system goes back to a typical JC model. However, in the presence of the optical parametric amplification, i.e., $G \neq 0$, all the new physical effects arise which we discuss in detail below. Before we discuss full numerical results, we like to highlight the physics behind the dressed-state crossing and the formation of avoided crossings.
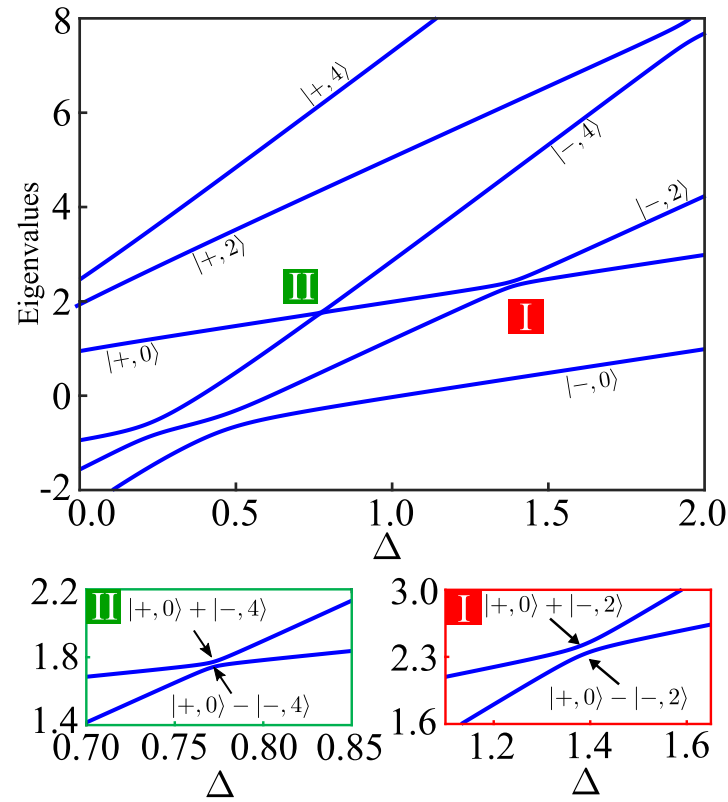

FIG. 2. Eigenvalues of the system against the detuning $\Delta$ with system parameters $g=1$ and $G=0.1$. Avoided level crossing involving $|+, 0\rangle$ and $|-, 2\rangle(|+, 0\rangle$ and $|-, 4\rangle)$ is indicated by label I (II). The lower two plots are the zoom-ins near the avoided level crossing regimes.

For $G=0$, as shown in Fig. 1(b), the eigenstates are the well-known doublets in the JC model, i.e., $| \pm, n\rangle \equiv(|e, n\rangle \pm$ $|g, n+1\rangle) / \sqrt{2}$ with eigenvalue $\lambda_{ \pm, n} \equiv(n+1) \Delta \pm \sqrt{n+1} g$. Obviously, the energy of eigenstate $|+, n\rangle$ increases, but the energy of eigenstate $|-, n\rangle$ decreases as the qubit-cavity coupling strength $g$ is enhanced. Thus, two eigenstates will cross at a specific detuning which is locked with the coupling strength. In the presence of the perturbation, i.e., the pump field, avoided level crossing between dressed states will occur, yielding quantum beats, entanglement, and the superposition state of even or odd Fock states. We present a simple, rather approximate discussion to bring out how the avoided crossings result.

Consider the dressed state $|+, 0\rangle$ and $|-, 2\rangle$ with energies $\lambda_{+, 0}=\Delta+g, \lambda_{-, 2}=3 \Delta-\sqrt{3} g$. These two dressed states cross at $\lambda_{+, 0}=\lambda_{-, 2}$ for $\Delta=(1+\sqrt{3}) g / 2$. Let us now assume that $G$ is small so that we can retain the coupling of $|+, 0\rangle$ and $|-, 2\rangle$ as illustrated by the red arrow in Fig. 1(b). Note that $|+, 0\rangle=(|e, 0\rangle+|g, 1\rangle) / \sqrt{2}$ and $|-, 2\rangle=(|e, 2\rangle-|g, 3\rangle) / \sqrt{2}$ are coupled in two different ways via the parametric drive which causes two photon transitions $|e, 0\rangle \stackrel{\sqrt{2} G}{\Longleftrightarrow}|e, 2\rangle$ and $|g, 1\rangle \stackrel{\sqrt{6} G}{\Longleftrightarrow}|g, 3\rangle$. This coupling results in two new energy levels which are now separated at $\Delta=(1+\sqrt{3}) g / 2$ by $2\left|\left\langle+, 0\left|G\left(a^{2}+a^{\dagger 2}\right)\right|-, 2\right\rangle\right|=$ $\sqrt{2}(\sqrt{3}-1) G$. This example demonstrates in a simple way how the parametric drive can produce avoided crossing of the dressed states $|+, 0\rangle$ and $|-, 2\rangle$. In Fig. 2 we show the behavior of the eigenvalues of the Hamiltonian, i.e., Eq. (1), as a function of $\Delta$ for $g=1, G=0.1$. We have kept the parametric coupling law so that we could work with truncated Hilbert space up to five photons. We display two avoided 

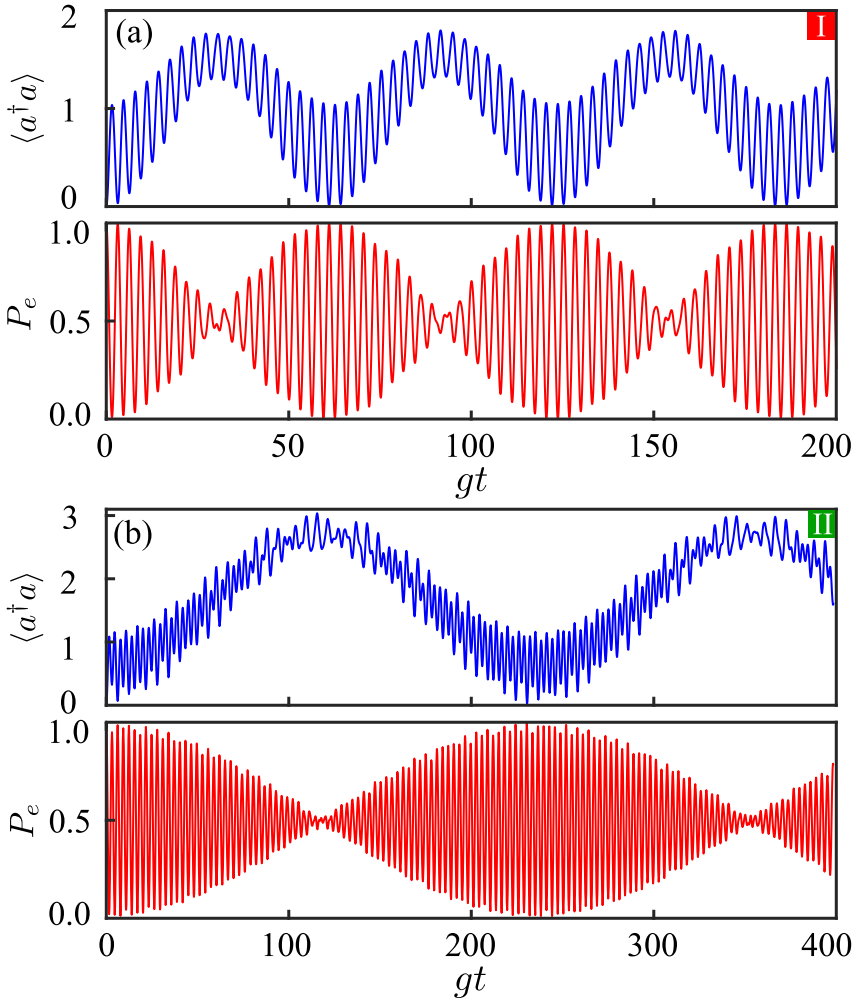

FIG. 3. Dynamical evolution of the mean photon number $\left\langle a^{\dagger} a\right\rangle$ (blue) and probability of finding qubit in the excited state $P_{e}$ (red) with initial condition $\Psi(0)=|e, 0\rangle$. Panels (a) and (b) correspond to the avoided level crossings I and II with the detuning $\Delta=$ $(1+\sqrt{3}) g / 2$ and $\Delta=(1+\sqrt{5}) g / 4$, respectively. Here, the system parameters are given by $g=1.0$ and $G=0.1$.

crossings involving dressed states $|+, 0\rangle$ and $|-, 2\rangle(|+, 0\rangle$ and $|-, 4\rangle)$.

\section{COHERENCES AND QUANTUM BEATS}

To quantify the above physical discussion, we numerically solve the Schrödinger equation $\partial \Psi / \partial t=-i H \Psi$ with $\Psi$ being the wave function of the system. In Fig. 3, we plot the mean photon number $\left\langle a^{\dagger} a\right\rangle$ and the probability of finding a qubit in the excited state $P_{e}$ as a function of the normalized evolution time $g t$. Here, we choose the detuning $\Delta=(1+$ $\sqrt{3}) g / 2$ (i.e., the avoided level crossing I) and other system parameters are the same as those used in Fig. 2. As shown in Fig. 3(a), there exist two different oscillation frequencies in time evolution of $\left\langle a^{\dagger} a\right\rangle$ and $P_{e}$. The faster oscillation results from the effective qubit-cavity coupling with a short period of $T_{1}=\pi / g$, while the slower oscillation originates from the energy difference at the avoided level crossing with a long period of $T_{2}=2 \pi /[(\sqrt{6}-\sqrt{2}) G]$ (the beat frequency is defined as $\left.f_{\text {beat }}=1 / T_{2}\right)$. At the quiet spots, the mean photon number reaches its maximum, but the population of the qubit excited state $P_{e}=0.5$. Likewise, at the avoided level crossing II [i.e., $\Delta=(1+\sqrt{5}) g / 4$ ], quantum beat behavior can also be observed in the cavity excitation spectrum and population of the qubit excited state. The corresponding beat frequency is much shorter than that for the avoided level crossing I.

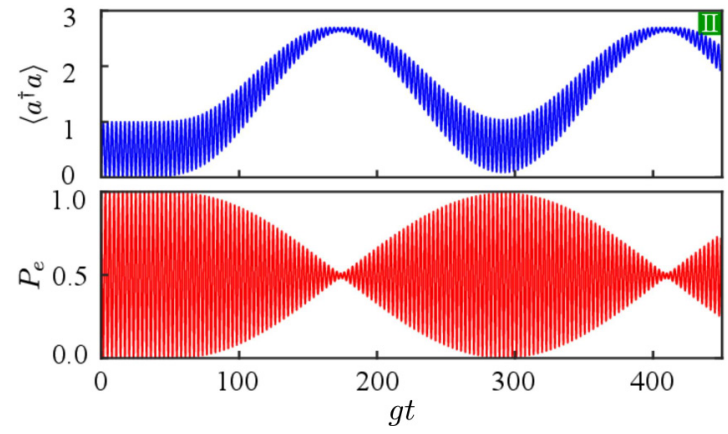

FIG. 4. Reduction of the multiphoton-induced noise in the mean photon number $\left\langle a^{\dagger} a\right\rangle$ (blue) and the population in the qubit excited state $P_{e}$ (red) by switching the parametric drive adiabatically. Here, the pump field is set as a time-dependent Gaussian profile, i.e., $G=G_{0}\left(1+\tanh \left[\left(t-T_{0}\right) /\left(2 \tau_{0}\right)\right]\right) / 2$ with $\tau_{0}=5$ and $T_{0}=50$. Other system parameters are the same as those used in Fig. 3(b).

It is to be noted that the beats result from the generated coherence between states $(|+, 0\rangle \pm|-, 4\rangle) / \sqrt{2}$. Compared with the results at the avoided level crossing $\mathrm{I}$, it is also noticed that there exists more noise in the cavity excitation spectrum due to the multiphoton process, i.e., involvement of states with photon numbers different from those involved in crossings. The chosen parameter $G / g=0.1$ corresponds to an OPA oscillating well below threshold value defined by $2 G<\Delta$, although recent investigations [38,39] use values of $G$ close to threshold value. We note that the OPA cavities have been extensively used in the context of the generation of squeezed light where these even have been operated near threshold (see, e.g., the classic work of Ref. [43]). In fact, the noise induced by the multiphoton process can be eliminated by switching the parametric drive adiabatically. To this end, we assume that the pump field has a time-dependent Gaussian profile, i.e., $G=G_{0}\left(1+\tanh \left[\left(t-T_{0}\right) /\left(2 \tau_{0}\right)\right]\right) / 2$ with $\tau_{0}=5$ and $T_{0}=50$. Other system parameters are the same as those used in Fig. 3(b). As shown in Fig. 4, the times for achieving quantum beats are delayed by $T_{0}$, but the periods of oscillation

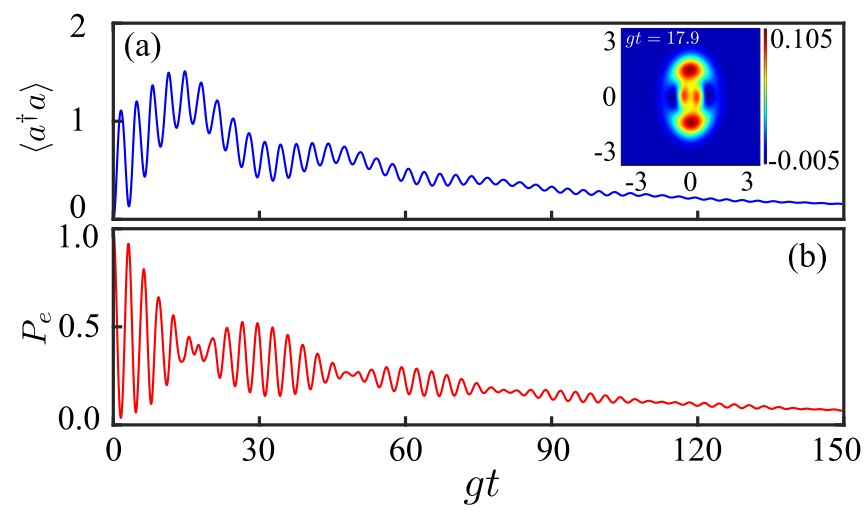

FIG. 5. Dynamical evolution of the (a) mean photon number $\left\langle a^{\dagger} a\right\rangle$ and (b) probability of finding qubit in the excited state $P_{e}$ for qubit and cavity dissipation rates $\gamma=\kappa=0.01$. Here, we choose $G=0.2, \Delta=1.430$ and other system parameters are the same as those in Fig. 6. The inset figure in panel (a) demonstrates the Wigner function of the cavity photon state at $g t=17.9$. 

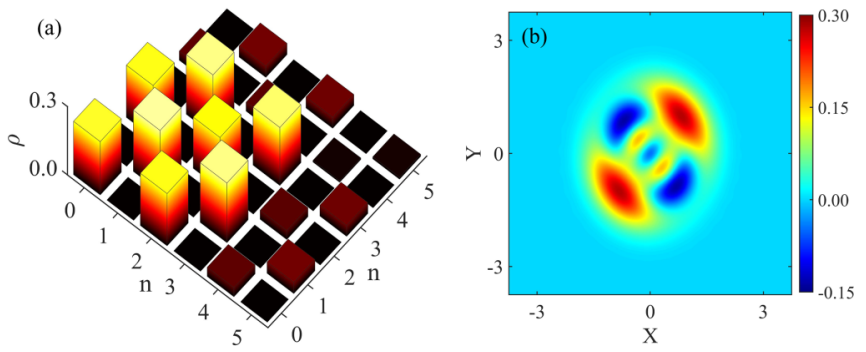

FIG. 6. The density matrix of the intracavity photon state (a) and the Wigner function (b) by numerically solving the Schrödinger equation. Here, the evolution time $g t \approx 31$ and the detuning is chosen as $\Delta=(1+\sqrt{3}) g / 2$, corresponding to the avoided level crossing I.

induced by the qubit-cavity coupling and quantum beats remain unchanged. Moreover, we find that the adiabatic driving can eliminate the noise in the cavity excitation spectrum and the spin evolution. Compared with the results in Fig. 3(b), the cavity field and population of the qubit excited state evolve more smoothly.

Note that we do not include cavity decay as typically quantum beats (and for that matter all coherence effects) are studied in the transient domain, i.e., during a period which is smaller than the cavity decay time. The domain that we consider is reached with superconducting qubits where $g / \kappa$ can be more than 200 [44]. In Fig. 5, we show the results for qubit and cavity dissipation rates $\gamma=\kappa=0.01, g=1, G=0.2$, and $\Delta=1.430$. Clearly, the quantum beats and catlike photon states [see the inset figure in panel (a)] survive even with these system parameters.

\section{EQUALLY WEIGHTED SUPERPOSITION OF EVEN OR ODD PHOTON NUMBER FOCK STATES}

Next, let us study the properties of photon states and the entanglement between qubit and cavity photons at the quiet spot of quantum beats, where the coherences of two dressed states are maximally established. Thus, the system is evolved into the superposition state of two dressed states, i.e., $\Psi \approx(|e\rangle \mid$ even $\rangle+\mathrm{e}^{i \phi}|g\rangle \mid$ odd $\left.\rangle\right) / \mathcal{A}$ with $\phi$ being the relative phase and $\mathcal{A}$ being the normalized coefficient. Here, the states $\mid$ even $\rangle$ and $\mid$ odd $\rangle$ denote the superposition of Fock states with even or odd photon numbers, respectively. For the crossing point I, the even and odd states can be approximately expressed as $\mid$ even $\rangle=(|0\rangle+i|2\rangle) / \sqrt{2}$ and $\mid$ odd $\rangle=$ $(|1\rangle+i|3\rangle) / \sqrt{2}$ according to the numerical solutions of the Schrödinger equation. Note that the parameter $G$ is chosen to be small so that Fock state superpositions with very high fidelity can be generated. In Fig. 6(a), we show the density matrix of the cavity photon state with system parameters $g=$ 1.0 and $t \approx 31 / \mathrm{g}$, where the superposition of states $|+, 0\rangle$ and $|-, 2\rangle$ are perfectly generated by the coherence. Here, the initial condition is chosen as $|e, 0\rangle$ and the detuning $\Delta$ is chosen near the avoided level crossing point I. As shown in Fig. 6(a), the probabilities of finding the Fock states $|0\rangle,|1\rangle,|2\rangle$, and |3) are approximately equal to each other. At the same time, the coherences between Fock states $|0\rangle$ and $|2\rangle(|1\rangle$ and $|3\rangle)$ denoted by diagonal bars in panel (a) reach their maximum. In Fig. 6(b), we show the corresponding Wigner function of the cavity photons, which exhibits a similar pattern of cat state, where the negativities (one in the origin and the other two in the second and fourth quadrants) of the Wigner function represent the nonclassicality of the cavity photon state and
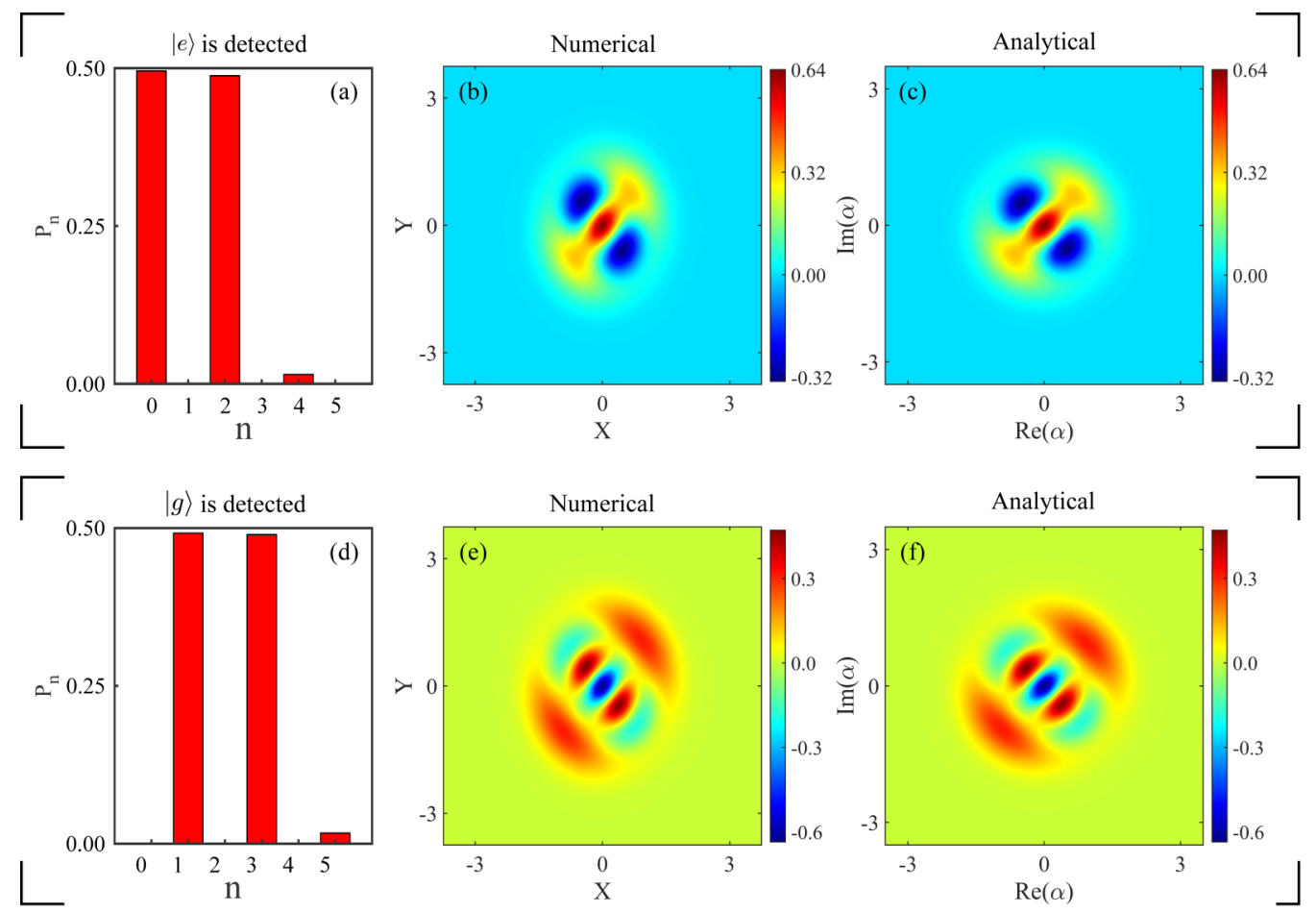

FIG. 7. (a), (d) Photon state distributions, (b), (e) the Wigner functions by solving the Schrödinger equation, and (c), (f) the analytical Wigner functions. Panels (a)-(c) correspond to the case where the qubit excited state $|e\rangle$ is detected. Panels (d)-(f) show the case of detecting the qubit ground state. Here, the evolution time $g t \approx 31$ and the detuning is $\Delta=(1+\sqrt{3}) g / 2$, corresponding to the avoided level crossing $\mathrm{I}$. 
the quantum interference fringes are denoted as a signature of coherence of Fock states. In the following, we will discuss how even or odd photon number Fock states result in the negativities in the Wigner function. It should be noted that the even and odd states that we generate are different from the cat states involving squeezed coherent states which are generated in the ultrastrong coupling regime $[33,38,39,45-47]$. With the coherence between dressed states, the qubit and the cavity photons are evolved into the maximally entangled state simultaneously. As shown in Fig. 7(a), if the qubit excited state $|e\rangle$ is detected, only Fock states with even photon numbers can be observed with equal weights. In this case, the Wigner function shown in panel (b) exhibits a similar pattern of even cat state, where two negativities occur in the second and fourth quadrants symmetrically. To show the physical mechanism more clearly, we analytically calculate the Wigner function $W(\alpha)=$ $\left(1 / \pi^{2}\right) \int d^{2} \lambda C_{w}(\lambda) \exp \left(\alpha \lambda^{*}-\alpha^{*} \lambda\right)$ by assuming the photon state $|\mathrm{ph}\rangle=\mid$ even $\rangle$. Here, the characteristic function $C_{w}(\lambda)=$ $\operatorname{Tr}[\rho \hat{D}(\lambda)]$ with density matrix $\rho=|\mathrm{ph}\rangle\langle\mathrm{ph}|$ and the displacement operator $\hat{D}(\lambda)=\exp \left(\lambda a^{\dagger}-\lambda^{*} a\right)$. Integrating the characteristic function $C_{w}$ by assuming $\lambda=x^{\prime}+i y^{\prime}$, one can obtain

$$
W(\alpha)=\frac{2}{\pi} e^{-2|\alpha|^{2}}\left[\left(1-2|\alpha|^{2}\right)^{2}+4 \sqrt{2} \operatorname{Re}(\alpha) \operatorname{Im}(\alpha)\right] .
$$

Note that the first term in the square brackets is always positive and the value of the Wigner function at the origin is always positive; the negativity of the Wigner function appears in the regime of $\operatorname{Re}(\alpha) \operatorname{Im}(\alpha)<-\left(1-2|\alpha|^{2}\right)^{2} /(4 \sqrt{2})<0$. As shown in Fig. 7(c), two negativities of this analytical Wigner function also appear in the second and fourth quadrants, which matches well with the numerical result. On the contrary, the cavity field will collapse to the superposition of odd photon number Fock states with equal weights [see Fig. 7(d)] if the qubit is measured in its ground state. The corresponding Wigner function is shown in panel (e) by solving the Schrödinger equation, which is similar to the odd cat state. Likewise, the Wigner function can also be calculated analytically by assuming the density matrix $\rho=\mid$ odd $\rangle\langle$ odd $|$, which yields

$$
\begin{aligned}
W(\alpha)= & \frac{1}{\pi} e^{-2|\alpha|^{2}}\left[-2\left(1-4|\alpha|^{2}\right)^{2}+8|\alpha|^{4}\left(1+\frac{4}{3}|\alpha|^{2}\right)\right. \\
& \left.+8 \sqrt{6}\left(\frac{4}{3}|\alpha|^{2}-1\right) \operatorname{Re}(\alpha) \operatorname{Im}(\alpha)\right] .
\end{aligned}
$$

Obviously, the analytical Wigner function shown in panel (f) agrees well with the numerical one. It is clear to see that the Wigner function at the origin is negative. We note that interference fringes in the Wigner functions [see panels (b) and (e)] prove the coherence between even or odd photon number Fock states, while the negativity of the Wigner function indicates the nonclassicality of superposition states of even or odd photon number Fock states.

The fidelity of the photon state can be evaluated according to the formula $\mathcal{F}_{\mathrm{ph}}=\left\langle\mathrm{ph}\left|\rho_{\mathrm{T}}\right| \mathrm{ph}\right\rangle$ with target density matrix of photons $\rho_{\mathrm{T}}=\mid$ even $\rangle\langle$ even $|+|$ odd $\rangle\langle$ odd $|$. Here, the state $|\mathrm{ph}\rangle$ represents the photon state by solving the master equation. As shown in Fig. 8, the fidelity of the photon state (blue) is always close to its maximum (i.e., $\mathcal{F}_{\mathrm{ph}}>0.95$ ). To show the

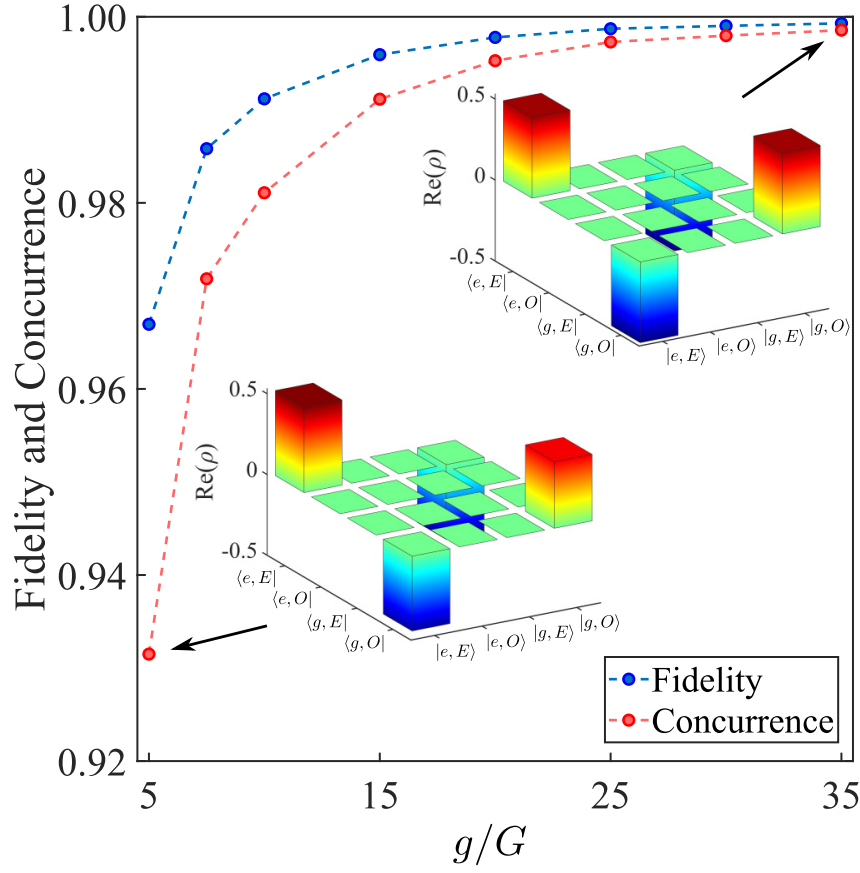

FIG. 8. The fidelity of the photon state (blue) and the concurrence of the system (red), representing the entanglement of the qubit and photons, are plotted as a function of the ratio $g / G$ with $G=0.1$. The inset plots demonstrate the real part of the density matrix of the system with $g=5 G$ and $35 G$, respectively. Here, $|E\rangle \equiv \mid$ even $\rangle$ $(|O\rangle \equiv \mid$ odd $\rangle$ ) represent the even (odd) photon number state.

entanglement between qubit and cavity photons quantitatively, we can define two artificial qubits, where the first qubit has two states $|e\rangle$ and $|g\rangle$, while the second qubit has two states $\mid$ even $\rangle$ and $\mid$ odd $\rangle$. Thus, the density matrix of the system can be reconstructed by using $|g\rangle \mid$ even $\rangle,|g\rangle \mid$ odd $\rangle,|e\rangle \mid$ even $\rangle$, and $|e\rangle \mid$ odd $\rangle$ as a set of new basis. Using the reconstructed density matrix $\rho^{\prime}$, the concurrence of the system (red) $C\left(\rho^{\prime}\right)$ [48], characterizing the entanglement of the qubit and photons is larger than 0.93 . We have also verified that the fidelity and concurrence do not change much in the neighborhood of the crossing point (see Fig. 9). As the qubit-cavity coupling strength increases, both the fidelity of photon state and the concurrence between qubit and photons will be significantly improved. For weak qubit-cavity coupling strength, although the photon state can be well produced the entanglement is not
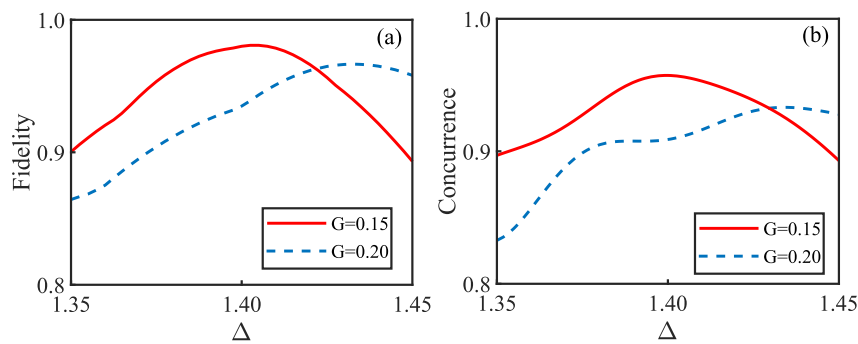

FIG. 9. The (a) fidelity and (b) concurrence versus the detuning for driving strength $G=0.15$ (solid curves) and 0.2 (dashed curves), respectively. Here, the evolution time is chosen near the quiet spot of the crossing point. 
very large since the energy transfer between qubit and cavity cannot be sufficiently established. To show this point, we take $g=5 G$ and plot the real part of the density matrix $\operatorname{Re}(\rho)$ in Fig. 8 (see inset figures). It is clear to see that the population in state $|e, E\rangle$ is slightly larger than that in state $|g, O\rangle$. For $g=35 G$, however, populations in state $|e, E\rangle$ and $|g, O\rangle$ have the same weights, corresponding to a maximum entanglement between qubit and cavity photons.

\section{CONCLUSIONS}

In conclusion, we have brought out many important consequences of the JC model when the cavity is incorporated by a parametric nonlinearity. The parametric nonlinearity produces avoided crossings between the dressed states of the JC model which otherwise cross at certain values of the detuning and the coupling of the qubit to the cavity field. At the avoided crossing one has well-defined coherence between the symmetric and the antisymmetric combinations of the dressed states which leads to quantum beats in physical parameters such as the excitation of the qubit. Another important feature arising from avoided crossing is the preparation of the equally weighted superpositions of the Fock states. More specifically we have shown the generation of superpositions of even (or odd) states. The fidelity of such a generation is more than
95\%. The challenging task of preparing equally weighted superpositions is achieved via the physics of the avoided crossings in the context of the cavity QED. We also demonstrate a very high degree of entanglement (concurrence more than $90 \%$ ) between the cavity field and the qubit. Our results use the dressed states of the JC model under conditions when the qubit is on resonance with the cavity frequency and the possible avoided crossings that can occur due to the switching on of a quantum drive. The case when the qubit frequency is detuned from the cavity frequency is a very different problem as the dressed-state structure is totally different and thus a subject of a new investigation. From the point of view of experiments, equality of the cavity and the qubit frequencies is not a limitation as cavities can be tuned very accurately.

\section{ACKNOWLEDGMENTS}

C.J.Z. and Y.P.Y. acknowledge the supports of National Nature Science Foundation (Grants No. 61975154 and No. 11874287) and National Key Research and Development Program of China (Grants No. 2021YFA1400600 and No. 2021YFA1400602). G.S.A. acknowledges the support of Air Force Office of Scientific Research (Award No. FA955020-1-0366).

L.L.P. and W.L. contributed equally to this work.
[1] W. D. Heiss and A. L. Sannino, Avoided level crossing and exceptional points, J. Phys. A: Math. Gen. 23, 1167 (1990).

[2] H. Eleuch and I. Rotter, Avoided level crossings in open quantum systems, Fortschr. Phys. 61, 194 (2013).

[3] C. Zener, Non-adiabatic crossing of energy levels, Proc. R. Soc. London, Ser. A 137, 696 (1932).

[4] L. D. Landau, Zur theorie der energieübertragung. II, Physics of the Soviet Union 2, 46 (1932).

[5] I. Rotter, Dynamics of quantum systems, Phys. Rev. E 64, 036213 (2001).

[6] J. R. Rubbmark, M. M. Kash, M. G. Littman, and D. Kleppner, Dynamical effects at avoided level crossings: A study of the Landau-Zener effect using Rydberg atoms, Phys. Rev. A 23, 3107 (1981).

[7] U. D. Rapol and V. Natarajan, Precise measurement of hyperfine intervals using avoided crossing of dressed states, Europhys. Lett. 60, 195 (2002).

[8] B. D. Patterson, Muonium states in semiconductors, Rev. Mod. Phys. 60, 69 (1988).

[9] D. M. Berns, M. S. Rudner, S. O. Valenzuela, K. K. Berggren, W. D. Oliver, L. S. Levitov, and T. P. Orlando, Amplitude spectroscopy of a solid-state artificial atom, Nature (London) 455, 51 (2008).

[10] J. Wiersig, Formation of Long-Lived, Scarlike Modes Near Avoided Resonance Crossings in Optical Microcavities, Phys. Rev. Lett. 97, 253901 (2006).

[11] E. D. Davis and W. D. Heiss, Random-phase approximation and broken symmetry, J. Phys. G 12, 805 (1986).

[12] K. J. Hornbostel, The application of light-cone quantization to quantum chromodynamics in one-plus-one dimensions, Ph.D. thesis, Stanford University, 1989.

[13] S. Haroche, M. Brune, and J. M. Raimond, From cavity to circuit quantum electrodynamics, Nat. Phys. 16, 243 (2020).
[14] G. S. Agarwal, Quantum Optics (Cambridge University Press, Cambridge, UK, 2012).

[15] J. J. Sanchez-Mondragon, N. B. Narozhny, and J. H. Eberly, Theory of Spontaneous-Emission Line Shape in an Ideal Cavity, Phys. Rev. Lett. 51, 550 (1983).

[16] G. S. Agarwal, Vacuum-Field Rabi Splittings in Microwave Absorption by Rydberg Atoms in a Cavity, Phys. Rev. Lett. 53, 1732 (1984).

[17] A. Boca, R. Miller, K. M. Birnbaum, A. D. Boozer, J. McKeever, and H. J. Kimble, Observation of the Vacuum Rabi Spectrum for One Trapped Atom, Phys. Rev. Lett. 93, 233603 (2004).

[18] P. Maunz, T. Puppe, I. Schuster, N. Syassen, P. W. H. Pinkse, and G. Rempe, Normal-Mode Spectroscopy of a SingleBound-Atom-Cavity System, Phys. Rev. Lett. 94, 033002 (2005).

[19] T. Yoshie, A. Scherer, J. Hendrickson, G. Khitrova, H. M. Gibbs, G. Rupper, C. Ell, O. B. Shchekin, and D. G. Deppe, Vacuum Rabi splitting with a single quantum dot in a photonic crystal nanocavity, Nature (London) 432, 200 (2004).

[20] N. F. Bell, R. F. Sawyer, R. R. Volkas, and Y. Y. Y. Wong, Generation of entangled states and error protection from adiabatic avoided level crossings, Phys. Rev. A 65, 042328 (2002).

[21] J. Karthik, A. Sharma, and A. Lakshminarayan, Entanglement, avoided crossings, and quantum chaos in an Ising model with a tilted magnetic field, Phys. Rev. A 75, 022304 (2007).

[22] E. Pachniak and S. A. Malinovskaya, Creation of quantum entangled states of Rydberg atoms via chirped adiabatic passage, Sci. Rep. 11, 1 (2021).

[23] S. De Nicola, A. A. Michailidis, and M. Serbyn, Entanglement View of Dynamical Quantum Phase Transitions, Phys. Rev. Lett. 126, 040602 (2021). 
[24] D. Bruß, N. Datta, A. Ekert, L. C. Kwek, and C. Macchiavello, Multipartite entanglement in quantum spin chains, Phys. Rev. A 72, 014301 (2005).

[25] R. G. Unanyan, M. Fleischhauer, N. V. Vitanov, and K. Bergmann, Entanglement generation by adiabatic navigation in the space of symmetric multiparticle states, Phys. Rev. A 66, 042101 (2002).

[26] S. Braungardt, A. Sen(De), U. Sen, and M. Lewenstein, Errorresistant distributed quantum computation in a trapped ion chain, Phys. Rev. A 76, 042307 (2007).

[27] G. S. Agarwal and W. Harshawardhan, Realization of trapping in a two-level system with frequency-modulated fields, Phys. Rev. A 50, R4465 (1994).

[28] B. Damski and W. H. Zurek, Adiabatic-impulse approximation for avoided level crossings: From phase-transition dynamics to Landau-Zener evolutions and back again, Phys. Rev. A 73, 063405 (2006).

[29] P. Huang, J. Zhou, F. Fang, X. Kong, X. Xu, C. Ju, and J. Du, Landau-Zener-Stückelberg Interferometry of a Single Electronic Spin in a Noisy Environment, Phys. Rev. X 1, 011003 (2011).

[30] S. Oh, Z. Huang, U. Peskin, and S. Kais, Entanglement, Berry phases, and level crossings for the atomic Breit-Rabi Hamiltonian, Phys. Rev. A 78, 062106 (2008).

[31] We note that Bimbard et al. have previously prepared superpositions of Fock states up to the two photons using parametric down conversion and remote state preparation [E. Bimbard, N. Jain, A. MacRae, and A. I. Lvovsky, Quantum-optical state engineering up to the two-photon level, Nat. Photonics 4, 243 (2010)]. However, in this scheme it is hard to produce the superposition of Fock states with equal weights.

[32] M. Tomka, O. El Araby, M. Pletyukhov, and V. Gritsev, Exceptional and regular spectra of a generalized Rabi model, Phys. Rev. A 90, 063839 (2014).

[33] R. Gutiérrez-Jáuregui and G. S. Agarwal, Probing the spectrum of the Jaynes-Cummings-Rabi model by its isomorphism to an atom inside a parametric amplifier cavity, Phys. Rev. A 103, 023714 (2021).

[34] C. J. Zhu, L. L. Ping, Y. P. Yang, and G. S. Agarwal, Squeezed Light Induced Symmetry Breaking Superradiant Phase Transition, Phys. Rev. Lett. 124, 073602 (2020).

[35] S. Huang and G. S. Agarwal, Enhancement of cavity cooling of a micromechanical mirror using parametric interactions, Phys. Rev. A 79, 013821 (2009).

[36] S. Huang and G. S. Agarwal, Normal-mode splitting in a coupled system of a nanomechanical oscillator and a parametric amplifier cavity, Phys. Rev. A 80, 033807 (2009).
[37] G. S. Agarwal and S. Huang, Strong mechanical squeezing and its detection, Phys. Rev. A 93, 043844 (2016).

[38] C. Leroux, L. C. G. Govia, and A. A. Clerk, Enhancing Cavity Quantum Electrodynamics Via Antisqueezing: Synthetic Ultrastrong Coupling, Phys. Rev. Lett. 120, 093602 (2018).

[39] W. Qin, A. Miranowicz, P.-B. Li, X.-Y. Lü, J.-Q. You, and F. Nori, Exponentially Enhanced Light-Matter Interaction, Cooperativities, and Steady-State Entanglement Using Parametric Amplification, Phys. Rev. Lett. 120, 093601 (2018).

[40] G. S. Agarwal and R. R. Puri, Cooperative behavior of atoms irradiated by broadband squeezed light, Phys. Rev. A 41, 3782 (1990).

[41] W. Qin, A. Miranowicz, H. Jing, and F. Nori, Generating LongLived Macroscopically Distinct Superposition States in Atomic Ensembles, Phys. Rev. Lett. 127, 093602 (2021).

[42] S. C. Burd, R. Srinivas, H. M. Knaack, W. Ge, A. C. Wilson, D. J. Wineland, D. Leibfried, J. J. Bollinger, D. T. C. Allcock, and D. H. Slichter, Quantum amplification of boson-mediated interactions, Nat. Phys. 17, 898 (2021).

[43] L.-A. Wu, H. J. Kimble, J. L. Hall, and H. Wu, Generation of Squeezed States by Parametric Down Conversion, Phys. Rev. Lett. 57, 2520 (1986).

[44] J. M. Fink, M. Göppl, M. Baur, R. Bianchetti, P. J. Leek, A. Blais, and A. Wallraff, Climbing the Jaynes-Cummings ladder and observing its nonlinearity in a cavity QED system, Nature (London) 454, 315 (2008).

[45] We note that a proposal for the preparation of a Fock state (and not a superposition state) has been made in the context of superconducting qubits and using the dispersive limit of the JC model [S. Krastanov, V. V. Albert, C. Shen, C. L. Zou, R. W. Heeres, B. Vlastakis, R. J. Schoelkopf, and L. Jiang, Universal control of an oscillator with dispersive coupling to a qubit, Phys. Rev. A 92, 040303 (2015)].

[46] Y. H. Chen, W. Qin, X. Wang, A. Miranowicz, and F. Nori, Shortcuts to Adiabaticity for the Quantum Rabi Model: Efficient Generation of Giant Entangled Cat States via Parametric Amplification, Phys. Rev. Lett. 126, 023602 (2021). This work reports an efficient way of time-dependent parametric drives to produce cat states.

[47] We note that P. Alsing, D. S. Guo, and H. J. Carmichael, Dynamic Stark effect for the Jaynes-Cummings system, Phys. Rev. A 45, 5135 (1992) have discussed nonclassical states of the JC model when the cavity is coherently driven. In contrast we derive the cavity by a quantum field ( $G$ term) which causes two photon transitions only.

[48] $C\left(\rho^{\prime}\right)=\max \left(0, \lambda_{1}-\lambda_{2}-\lambda_{3}-\lambda_{4}\right)$. Here $\lambda_{i}$ 's are square roots of eigenvalues of the matrix $\rho^{\prime} \tilde{\rho}^{\prime}$ in decreasing order. $\tilde{\rho}^{\prime}\left(\rho^{\prime *}\right)$ means the transpose (complex conjugation) of $\rho^{\prime}$. 